\title{
A new phase in oxide interface research
}

Electronic phase separation at the LaAlO(3)/SrTiO(3) interface

Authors: Ariando et. al.

Nature Communications 2 Article 188 (2011).

Coexistence of Superconductivity and Ferromagnetism in Two Dimensions

Authors: D. A. Dikin, M. Mehta, C. W. Bark, C. M. Folkman, C. B. Eom, and V. Chandrasekhar.

Phys. Rev. Lett. 107056802 (2011).

Coexistence of magnetic order and two-dimensional superconductivity at $\mathrm{LaAlO}_{3} / \mathrm{SrTiO}_{3}$ interfaces

Authors: Lu Li, C. Richter, J. Mannhart and R. C. Ashoori

Nature Physics, doi 10.1038/nphys2080.

Direct Imaging of the coexistence of ferromagnetism and superconductivity at the $\mathrm{LaAlO}_{3} / \mathrm{SrTiO}_{3}$ interface

Authors: J. A. Bert, B. Kalisky, C. Bell. M. Kim, Y. Hikita, H. Y. Hwang and K. Moler Nature Physics, doi 10.1038/nphys2079

\section{Recommended with a Commentary by A. J. Millis Department of Physics, Columbia University}

Naturally occurring transition metal oxides exhibit fascinating and potentially useful correlated electron properties including high temperature superconductivity, 'colossal' magnetoresistance and 'Mott' (correlation-driven) metal-insulator transitions, but these typically occur at inconvenient regimes of temperature, pressure or other parameters, or in materials which cannot be easily synthesized or interfaced with other materials to create devices. Hence the hope that an electron gas formed at an appropriately designed oxide interface will exhibit new electronic properties, or at least optimizable versions of known ones.

The papers discussed here suggest that this dream has moved one step closer to reality. The papers study the (001) $\mathrm{LaAlO}_{3} / \mathrm{SrTiO}_{3}$ interface, which has emerged as a model system in part because the two component insulators, $\mathrm{LaAlO}_{3}$ and $\mathrm{SrTiO}_{3}$ are well understood and widely used in technology. $\mathrm{LaAlO}_{3}$ is a strong insulator; even the electronic states 
associated with defects are localized. In $\mathrm{SrTiO}_{3}$ low levels of impurities or oxygen defects can lead to conduction and (at very low temperature) superconductivity. Both $\mathrm{LaAlO}_{3}$ and $\mathrm{SrTiO}_{3}$ crystalize in slightly distorted versions of the cubic $\mathrm{ABO}_{3}$ perovskite. The label (001) indicates the orientation of the interface relative to one of the principal axes of this structure. The (001) $\mathrm{LaAlO}_{3} / \mathrm{SrTiO}_{3}$ interface is polar: electrostatic considerations [1] imply that in the absence of chemical defects an interface between two semi-infinite materials would sustain an interfacial electron gas, of areal charge density 0.5 electrons per interfacial unit cell $\left(\sim 3 \times 10^{14} / \mathrm{cm}^{2}\right)$, much higher than can be obtained in conventional semiconductor interfaces and potentially high enough to produce interesting many-body physics.

Band theory suggests that the electrons would reside on states derived from $T i$ d-orbitals [2] and that the strong correlation effects associated with these states lead to ferromagnetism [3] and charge order [4]. But what happens in reality? In an initial phase of research, $\mathrm{LaAlO}_{3} / \mathrm{SrTiO}_{3}$ interfaces were fabricated [5], and superconductivity was observed [6] as (in differently prepared samples) was evidence of magnetism [7]. However, the superconducting properties of the interface system are similar in many respects to the superconductivity known to occur in lightly doped $\mathrm{SrTiO}_{3}$ while the original evidence for magnetism involved relatively weak effects on the temperature and magnetic field dependence of the electrical conductivity which could be blamed on magnetic defects. Dependence of properties on sample growth conditions suggests that the phases might be due to growth-related defects and not to an interfacial electron gas. Further, the carrier density (as determined from electrical transport measurements) was initially found to be inconsistent with the electrostatic mechanism because it was much too small, and had the wrong dependence on thickness of the $\mathrm{LaAlO}_{3}$ layer [8]. However, a subsequent tour-de-force high energy photoemission experiment by Sing and collaborators was able to measure the total interface electron density [9], finding results consistent with the electrostatic mechanism. The inference is that much of the added charge is not mobile and indeed resides within $\sim 1$ unit cell of the interface.

We are now discuss the recent work. Ariando and collaborators showed that magnetism occurs in interfaces grown at high oxygen partial pressure, with evidence of ordering persisting up to room temperature. As the oxygen partial pressure during growth is reduced, the size of the moment is found to decrease, becoming very small at the growth pressures which previous workers had found to optimize the superconducting properties. Dikin et al performed low temperature transport measurements which reveal both magnetoresistive 
effects associated with ferromagnetism and (at low temperatures and low applied fields) the zero resistance characteristic of superconductivity, suggesting that the two phenomena coexist in the same sample. The most recent two papers provide more direct evidence of the magnetism and its coexistence with superconductivity. Li et. al. used torque magnetometry (essentially a mechanical measurement of the force exerted on a magnet by an applied field) to determine quantitatively the field and temperature dependence of the magnetization finding a ferromagnetic state with magnetization values corresponding to aligning about $2 / 3$ of the density of electrons expected from the electrostatic arguments (these values are somewhat smaller than Ariando et al found in samples grown under similar conditions). Resistivity measurements show that the sample is also superconducting. Bert et al used a scanning squid magnetometer to image the coexisting magnetic and superconducting states on a few micron length scale. The superconductivity is inhomogeneous, with the superconducting regions occupying only a small fraction of the areas measured. The magnetic regions are similarly inhomogeneous, with patches of micron-scale ferromagnetism coexisting with a higher density of much smaller-scale domains of fluctuating local magnetic moments. Remarkably, the total density of magnetic moments (including both fluctuating moments and those in observable ferromagnetic domains) was found to be approximately equal to the 0.5 electrons per unit cell implied by electrostatics.

The results are all, broadly speaking, consistent and consistent with the finding of Sing et. al [9]. They strongly suggest that it is these states that give rise to the magnetization. Confidence is increased because the publications are based on samples grown in four different labs. The findings favor a picture in which electrostatic arguments correctly predict the electron density, but in which most of the electrons reside in localized (and quite probably magnetic) states near the interface, while a small density (perhaps $\sim 10 \%$ ) reside father from the interface in mobile states which may become superconducting. But there are many questions. The relation between oxygen partial pressure during growth, annealing protocol and the occurrence of magnetism and of superconductivity needs to be unravelled. While the general picture of a polar discontinuity-induced electron gas seems to have been confirmed, the degree to which defects permit the existence of mobile electrons far from the interface remains unresolved. Quantitative discrepancies between the different experiments remain. The cause (disorder or a many-body effect) for apparent localization of the high density of carriers at the interface must be determined. The simultaneous occurrence of 
ferromagnetism and superconductivity (two phases normally believed to be incompatible) is already attracting theoretical attention [10] although the observed spatial inhomogeneity may indicate that the two phases simply live apart from each other.

But most importantly, the confirmation in different ways by Ariando et al, Dikin et. al., Li et al and Bert et al of the ferromagnetism first hinted at by Brinkman et al [7] shows that an oxide interface can exhibit an electronic phase (ferromagnetism) which does not occur in either component material separately is an existence proof of new phenomena at an oxide interface, and should stimulate a wider-ranging search for other novel interface-related effects.

[1] J. Mannhart, D. A. Blank, H. Y. Hwang, A. J. Millis, and J. M. Triscone, Bulletin of the Materials Research Society 33, 1027 (2008).

[2] Z. S. Popovic and S. Satpathy, Phys. Rev. Lett. 94, 176805 (2005).

[3] S. Okamoto, A. J. Millis, and N. A. Spaldin, Phys. Rev. Lett. 97, 056802 (2006).

[4] R. Pentcheva and W. E. Pickett, Phys. Rev. Lett. 99, 016802 (2007).

[5] A. Ohtomo and H. Hwang, NATURE 427, 423 (2004), ISSN 0028-0836.

[6] N. Reyren, S. Thiel, A. D. Caviglia, L. F. Kourkoutis, G. Hammerl, C. Richter, C. W. Schneider, T. Kopp, A.-S. Ruetschi, D. Jaccard, et al., Science 317, 1196 (2007), http://www.sciencemag.org/cgi/reprint/317/5842/1196.pdf, URL http://www . sciencemag. org/cgi/content/abstract/317/5842/1196.

[7] A. Brinkman, M. Huijben, M. van Zalk, J. Huijben, U. Zeitler, J. C. Maan, W. G. van der Wiel, G. Rijnders, D. H. A. Blank, and H. Hilgenkamp, Nature Materials 6, 493 (2009), URL http://www.nature.com/nmat/journal/v6/n7/suppinfo/nmat1931_S1.html.

[8] S. Thiel, G. Hammerl, A. Schmehl, C. W. Schneider, and J. Mannhart, Science 313, 1942 (2006), http://www.sciencemag.org/cgi/reprint/313/5795/1942.pdf, URL http://www . sciencemag. org/cgi/content/abstract/313/5795/1942.

[9] M. Sing, G. Berner, K. Goß, A. Müller, A. Ruff, A. Wetscherek, S. Thiel, J. Mannhart, S. A. Pauli, C. W. Schneider, et al., Phys. Rev. Lett. 102, 176805 (2009).

[10] K. Michaeli, A. Potter, and P. A. Lee, arXiv:1107.4352 (2011). 